\title{
High-energy scissors mode
}

\author{
R. Nojarov, ${ }^{*}$ Amand Faessler ${ }^{\dagger}$ and M. Dingfelder ${ }^{\ddagger}$ \\ Institut für Theoretische Physik, Universität Tübingen, \\ Auf der Morgenstelle 14, D-72076 Tübingen, Germany
}

(28 November 1994)

\begin{abstract}
All the orbital M1 excitations, at both low and high energies, obtained from a rotationally invariant QRPA, represent the fragmented scissors mode. The high-energy M1 strength is almost purely orbital and resides in the region of the isovector giant quadrupole resonance. In heavy deformed nuclei the highenergy scissors mode is strongly fragmented between 17 and $25 \mathrm{MeV}$ (with uncertainties arising from the poor knowledge of the isovector potential). The coherent scissors motion is hindered by the fragmentation and $B(M 1)<$ $0.25 \mu_{N}^{2}$ for single transitions in this region. The $\left(e, e^{\prime}\right)$ cross sections for excitations above $17 \mathrm{MeV}$ are one order of magnitude larger for E2 than for M1 excitations even at backward angles.
\end{abstract}

24.30.Cz, 25.30.Fj, 21.60.Jz, 27.70.+q

Typeset using REVTEX

\footnotetext{
*Permanent address: Institute for Nuclear Research and Nuclear Energy, Bulgarian Academy of Sciences, BG-1784, Sofia, Bulgaria. Electronic address: nojarov@mailserv.zdv.uni-tuebingen.de

†faessler@mailserv.zdv.uni-tuebingen.de

‡dingfelder@mailserv.zdv.uni-tuebingen.de
} 


\section{INTRODUCTION}

The nature of the orbital magnetic dipole (M1) strength in deformed nuclei is still a controversial matter. The low-lying orbital excitations with $K^{\pi}=1^{+}$have been interpreted [1] as isovector rotational vibrations, i. e. as a manifestation of a weakly collective scissors mode [2]. A strongly collective scissors state was predicted by the classical picture of the Two-Rotor Model [3], assuming that neutrons and protons perform out-of-phase rotational oscillations around an axis perpendicular to the nuclear symmetry axis. The fragmentation of the M1 strength over a broad energy region, found experimentally between 2 and $4 \mathrm{MeV}$ in heavy deformed nuclei (e. g. Refs. 四) and present in microscopic theoretical calculations, hinders the comparison with the simple classical picture. It has been shown earlier [1,5] that the energy and the amount of the high-lying M1 strength, corresponding to $\Delta \mathcal{N}=2$ excitations, are strongly dependent on the type of the residual quadrupole-quadrupole (or non-spin) interaction used in microscopic calculations within the quasiparticle random-phase approximation (QRPA).

The above interpretation in terms of scissors mode is supported by some theoretical works [6 10] and questioned by others [11 15]. The scissors mode is found in Refs. [13 15] at high energy, $\mathrm{E}_{\text {theor }} \approx 22-24 \mathrm{MeV}$, as a part of the isovector giant quadrupole resonance (IVGQR). These three works use Landau-Migdal interactions in QRPA. Similar conclusions are obtained in QRPA with separable forces [16], where the IVGQR lies at $\mathrm{E}_{\text {theor }} \approx 27 \mathrm{MeV}$ and overlaps $40 \%$ with the scissors mode. It was shown 14 that the collective hydrodynamical model predicts a high-energy scissor mode, but the low-lying state is spurious. However, after taking additionally the nuclear elasticity within the same classical model into account, a low-energy mode was obtained as well [15]. The isovector rotor model [17] predicts the appearance of the scissors mode at both low and high energies.

We are going to discuss in Sec. III the isovector coupling of this interaction, which influences strongly the high-lying orbital M1 strength. An isoscalar symmetry restoring interaction is introduced in Sec. II, together with an isovector interaction. The E2 strength 
distribution is studied in Sec. III in relation to the isovector coupling constant. The highenergy M1 strength and related $\left(e, e^{\prime}\right)$ cross sections are discussed in Sec. IV. The conclusions are summarized in Sec. V.

\section{DECOUPLED ISOVECTOR INTERACTION}

The deformed mean field $\mathbf{H}_{0}$ is obtained in our formalism [1, 18] by diagonalizing an axially-symmetric Woods-Saxon potential. Electric and magnetic transition operators are treated on equal footing by introducing a signature $m= \pm 1$ [1], corresponding to the directions $x$ and $y$, indistinguishable in the intrinsic frame. For instance, the total angular momentum operator, $\mathbf{J}_{t} \equiv \mathbf{J}(m, t)$, defined by Eq. (4) of [5], has quasiparticle (q.p.) matrix elements $j_{k i}^{t} \equiv j(k i, m t)$ [19]. The isospin index $t$ denotes neutrons or protons. We shorten the notations in the following by omitting, wherever possible, the signature index $m$, which is of technical interest only.

Following the procedure of Pyatov [20], we construct in the quasiboson approximation operators of quadrupole type $\mathbf{F}$. They are used to define the isoscalar interaction $\mathbf{H}_{S}$ [1, 5], which restores the rotational symmetry of the mean field $\mathbf{H}_{0}$, violated by the deformation:

$$
\begin{array}{r}
\mathbf{F}_{t} \equiv \mathbf{F}(m, t)=\left[\mathbf{H}_{0}, \mathbf{J}_{t}\right], \\
\mathbf{F}_{S}=\mathbf{F}_{n}+\mathbf{F}_{p}, \quad \mathbf{F}_{V}=\mathbf{F}_{n}-\mathbf{F}_{p}, \\
\mathbf{H}_{S}=-\frac{k_{S}}{2} \sum_{m} m \mathbf{F}_{S}^{2}(m),
\end{array}
$$

Under the assumption for a simple quadrupole deformation $\mathbf{Q}_{20}$ only, as in the case of the Nilsson potential, the operators $\mathbf{F}_{S}(m)$ (1) are proportional to the quadrupole operators $\mathrm{Q}_{2, \pm 1}$

A separable residual interaction of the general form,

$$
\begin{array}{r}
\mathbf{H}_{F F}=-\frac{1}{2} \sum_{m} m\left[k_{n n} \mathbf{F}_{n}^{2}(m)+k_{p p} \mathbf{F}_{p}^{2}(m)\right. \\
\left.+2 k_{n p} \mathbf{F}_{n}(m) \mathbf{F}_{p}(m)\right],
\end{array}
$$


can be decomposed into a sum of isoscalar, isovector, and coupling terms with the corresponding coupling constants,

$$
\begin{array}{r}
k_{0}=\frac{1}{4}\left(k_{n n}+k_{p p}+2 k_{n p}\right), \\
k_{1}=\frac{1}{4}\left(k_{n n}+k_{p p}-2 k_{n p}\right), \\
k^{\prime}=\frac{1}{2}\left(k_{n n}-k_{p p}\right), \quad r^{\prime}=k_{1} / k_{0} .
\end{array}
$$

The isovector operator $\mathbf{F}_{V}$ (1) is obtained from the isovector angular momentum $\mathbf{J}_{n}-\mathbf{J}_{p}$. However, it is more convenient to construct a relative isovector interaction $\mathbf{H}_{R}$ and the coupling $\mathbf{H}_{C}$ with the isoscalar term (1) by introducing a relative angular momentum $\mathbf{J}_{R}$ :

$$
\begin{array}{r}
\mathbf{J}_{R}=\sqrt{\frac{X_{p}}{X_{n}}} \mathbf{J}_{n}-\sqrt{\frac{X_{n}}{X_{p}}} \mathbf{J}_{p}, \quad X_{t}=\left\langle\left[\mathbf{J}_{t}^{\dagger}, \mathbf{F}_{t}\right]\right\rangle, \\
\mathbf{F}_{R} \equiv \mathbf{F}_{R}(m)=\left[\mathbf{H}_{0}, \mathbf{J}_{R}(m)\right], \\
\mathbf{H}_{R}=-\frac{k_{R}}{2} \sum_{m} m \mathbf{F}_{R}^{2}(m), \\
\mathbf{H}_{C}=-\frac{k_{C}}{2} \sum_{m} m \mathbf{F}_{R}(m) \mathbf{F}_{S}(m) .
\end{array}
$$

It is easy to show that the relative isovector interaction $\mathbf{H}_{R}$ is rotationally invariant and its coupling constant $k_{R}$ remains a free parameter. This interaction is slightly more symmetric than that used in Ref. [12], but the difference is insignificant. The condition for rotational symmetry provides the same value for the isoscalar constant $k_{S}$ as in Eq. (9) of [5] and closes the coupling $\mathbf{H}_{C}$ between the isoscalar and relative channels:

$$
\begin{array}{r}
{\left[\mathbf{H}_{0}+\mathbf{H}_{S}+\mathbf{H}_{R}+\mathbf{H}_{C}, \mathbf{J}\right]=0,} \\
\Longrightarrow k_{S}=1 / X, k_{C}=0, \quad X=X_{n}+X_{p} .
\end{array}
$$

Thus, the use of relative interaction enables the decoupling of the two channels and the symmetry-restoring procedure [1,5] is no more necessary, since the hamiltonian (5) is already rotationally invariant.

We introduce further a spin-spin residual interaction $\mathbf{H}_{S S}$ [18, which is always rotationally invariant and does not change the coupling constants (5). The latter can be related to 
the coupling strengths of the quadrupole interaction in the general form (2)). Thus, our q.p. model hamiltonian, used in the present calculations, has the following form:

$$
\begin{array}{r}
\mathbf{H}=\mathbf{H}_{0}+\mathbf{H}_{F F}+\mathbf{H}_{S S}, \\
k_{n n}=k_{S}\left(1+r \frac{X_{p}}{X_{n}}\right), \quad k_{p p}=k_{S}\left(1+r \frac{X_{n}}{X_{p}}\right), \\
k_{n p}=k_{S}(1-r), \quad r=\frac{k_{R}}{k_{S}},
\end{array}
$$

where $k_{S}$ is calculated microscopically (5) and the ratio $r$ is treated as a free parameter to be discussed in the next section. The relationships with the constants $k_{0}, k_{1}$, and $k^{\prime}$ are obtained upon insertion of (6) into (3). The constants of the spin-spin interaction,

$$
c(+) A=200 \mathrm{MeV}, \quad c(-)=-0.5 c(+),
$$

are derived from nuclear matter calculations in the way described in Ref. [21]. The deformation-independent Woods-Saxon parameters are taken from Ref. [22]. When applied to other rare-earth [23] and actinide [21] nuclei, this parametrization has lead to a good agreement with experimental data for single M1 transitions at low energy, observed in $\left(e, e^{\prime}\right)$ and $\left(\gamma, \gamma^{\prime}\right)$ experiments. The energy distribution of the spin-flip M1 strength between 6 and $10 \mathrm{MeV}$, determined by inelastic proton scattering, has also been reproduced in [21,23].

The QRPA equations of motion are solved for $K^{\pi}=1^{+}$excitations in the intrinsic frame using the model hamiltonian (6). We present here as an example the results for ${ }^{160} \mathrm{Gd}$, obtained with deformations $\beta_{2}=0.26, \beta_{4}=0.025$ and pairing gaps $\Delta_{n}=0.8 \mathrm{MeV}, \Delta_{p}=$ 1.1 MeV. Expressions for M1 and E2 transition probabilities in terms of RPA amplitudes are given in Ref. [四]. The $\mathrm{B}(\mathrm{M} 1)$ values for transitions to $I^{\pi} K=1^{+} 1$ states are calculated with bare orbital and effective spin gyromagnetic factors, $g_{s}=0.7 g_{s}^{\text {free }}$. The $\mathrm{B}(\mathrm{E} 2)$ values for transitions to the corresponding $I^{\pi} K=2^{+} 1$ states are obtained without introducing effective charges, i. e. they are of purely proton nature.

Our attention shall be focused in the next section on the ratio $r$ (ब), determining the strength of the isovector spin-independent interaction. 


\section{ISOVECTOR COUPLING CONSTANT}

The energy distribution of the E2 strength with $K=1$ is shown in Fig. 1 as histograms for three different values of the isovector coupling constant $k_{R}$ (6). The latter is fixed by the ratio $r=k_{R} / k_{S}$, because the isoscalar constant $k_{S}$ is determined microscopically from (5). The lower bump has a peak at about $11 \mathrm{MeV}$, which is smeared out by the histogram. It represents the $K=1$ component of the isoscalar giant quadrupole resonance (ISGQR), observed by inelastic electron scattering [24]. Its position, determined by the isoscalar constant $k_{S}$, agrees with the prediction of the vibrating potential model [25],

$$
E(21 ; i s) \approx \frac{63}{A^{1 / 3}}\left(1-\frac{\delta}{6}\right) \mathrm{MeV} .
$$

The summed contribution of the $K= \pm 1$ components to the classical isoscalar and isovector energy-weighted (e.w.) sums are [26]

$$
\begin{array}{r}
S(E 21 ; i s)_{C}=S(E 21)_{C} Z / A, \\
S(E 21 ; i v)_{C}=S(E 21)_{C} N / A, \\
S(E 21 ; i s)_{C}+S(E 21 ; i v)_{C}=S(E 21)_{C}, \\
S(E 21)_{C}=\frac{5 e^{2} \hbar^{2}}{2 \pi m} Z\left\langle r^{2}\right\rangle_{p} .
\end{array}
$$

We calculate microscopically the mean-square-radius, $\left\langle r^{2}\right\rangle_{p}=27.3 \mathrm{fm}^{2}$, and obtain with this value from (9) the classical e.w. sum $S(E 21)_{C}=57573 e^{2} \mathrm{fm}^{4} \mathrm{MeV}$. The RPA isoscalar E2 e.w. strength between 9 and $11 \mathrm{MeV}$, shown in the middle plot of Fig. 1, is $S(E 21 ; 9-11 \mathrm{MeV})_{\mathrm{th}}=12215 e^{2} f m^{4} \mathrm{MeV}$, i.e. only $53 \%$ from the classical isoscalar e.w. sum $S(E 21 ; i s)_{C}=23029 e^{2} \mathrm{fm}^{4} \mathrm{MeV}$ are exhausted. This result corroborates with the experimental data for other heavy deformed nuclei [24]. However, no strength is missing, since the total RPA e.w. strength, $S(E 21 ; 0-30 \mathrm{MeV})_{\mathrm{th}}=57272 e^{2} \mathrm{fm}^{4} \mathrm{MeV}$, exhausts $99.5 \%$ of the above classical value $S(E 21)_{C}$. One should note further that the decomposition (9) of the classical E2 strength into isoscalar and isovector parts is a rather crude approximation, because it does not take into account the redistribution of these two components caused by the residual interactions. 
It is seen from Fig. 1 that the low energy E2 strength distribution and the position of the ISGQR are rather insensitive to the isovector residual interaction. The latter is repulsive and concentrates the isovector strength in the higher-lying bump, consisting of $\Delta \mathcal{N}=2$ excitations. The non e.w. E2 strength at high energy decreases for a stronger isovector interaction, because the e.w. E2 strength is conserved in the three plots of Fig. 1. This is due to the fact that the interaction (2) redistributes the E2 strength, but does not contribute to the E2 e.w. sum rule. The latter is determined by the mean field and the spin-spin interaction [18], which are fixed in the three cases of Fig. 1.

The isovector coupling constant $k_{R}$ could be determined from the position of the second maximum, representing the isovector giant quadrupole resonance (IVGQR). Experimental data on it for heavy deformed nuclei are scarce [27]. The measured $\left(e, e^{\prime}\right)$ spectrum is usually fitted by a number of gaussians. That one, lying closest to the theoretically expected energy 25],

$$
E(21 ; i v) \approx \frac{141}{A^{1 / 3}}\left(1-\frac{\delta}{6}\right) \mathrm{MeV}
$$

is identified with the IVGQR. The prediction (10) results from evaluation of the isovector coupling constant within the collective model. The main uncertainty of this procedure originates from the isovector potential $V_{1}$, which is estimated by relating the isovector density with the potential asymmetry energy

$$
\begin{array}{r}
\frac{V_{1}}{8} \int \frac{\left(\rho_{n}-\rho_{p}\right)^{2}}{\rho} \mathrm{d} \mathbf{r}=K_{a s} \frac{(N-Z)^{2}}{A}, \\
V_{1}=8 K_{a s} .
\end{array}
$$

Using a recent empirical value, $K_{V}=30.6 \mathrm{MeV}$ [28], for the volume asymmetry energy from the Bethe-Weizsäcker formula, and subtracting the kinetic energy $b_{k i n}=2 K_{k i n}=25 \mathrm{MeV}$ [29], one obtains $K_{a s}=K_{V}-K_{k i n} \approx 18 \mathrm{MeV}$. The resulting isovector potential $V_{1}=144$ $\mathrm{MeV}$ (12) is a little bit larger than the previous estimate of $130 \mathrm{MeV}$ [29], based on a smaller value of $K_{V}$.

The identity (12) follows from (11) under the assumption that the integral in the l.h.s. of (11) is equal to $(N-Z)^{2} / A$. This is true for constant neutron and proton densities 
within a sphere with a sharp surface. When the integral is calculated with more realistic Woods-Saxon densities and the isovector potential is determined from (11), instead of (12), we obtain $V_{1}=67.5 \mathrm{MeV}$ for ${ }^{160} \mathrm{Gd}$. Test calculations for several more rare-earth nuclei have lead to values close to $68 \mathrm{MeV}$.

The ratio between the isoscalar and isovector coupling constants of the quadrupole interaction can be calculated within the collective model 225. Using our microscopic mean square radius for ${ }^{160} \mathrm{Gd}$, the above isovector potential $V_{1}=67.5 \mathrm{MeV}$, and an oscillator constant $\hbar \omega_{0} A=41 \mathrm{MeV}$, we obtain for this ratio $r^{\prime}=k_{1} / k_{0}=-1.5$. However, as discussed in Sec. II, the operators F (1) do not coincide with the quadrupole operators. Moreover, the isoscalar coupling constant $k_{S}$ is already determined from the rotational invariance (6). The isovector constant $k_{R}$ can be evaluated in our case following the procedure of Ref. 25] for the operator $\mathbf{F}$ :

$$
\begin{aligned}
k_{R}=-\frac{V_{1}}{8}\left[\int \mathbf{F}^{\dagger} \rho \mathbf{F} d \mathbf{r}\right]^{-1}= & -\frac{V_{1}}{8}\left\langle\mathbf{F} \mathbf{F}^{\dagger}\right\rangle^{-1}, \\
\left\langle\mathbf{F F}^{\dagger}\right\rangle & =2 \sum_{i k} E_{i k}^{2} j_{i k}^{2},
\end{aligned}
$$

where the summation runs over neutrons and protons. An additional factor of $1 / 2$ is present in (13) in comparison with [25], since our coupling constants (2.,3) are two times smaller than usual. This is due to the $m$-symmetrization of the interaction $\mathbf{H}_{F F}$ (2, (6). Using $V_{1}=67.5$ $\mathrm{MeV}$, one obtains from (5.13) the ratio $r=k_{R} / k_{S}=-1.67$, consistent with the above value of $r^{\prime}$ for a quadrupole interaction.

These numbers contain still rather large uncertainties. Corrections to the isovector potential could arise, e. g. from the surface asymmetry energy. It contributes even to the IVGQR [30], regarded as a predominantly volume oscillation. Further renormalization effects [31] can lead even to attractive n-n and p-p quadrupole interactions with $r^{\prime}=-0.6$. Thus, the theoretical estimates of the isovector coupling constant are not accurate enough and only the experimental data on the IVGQR could decide on this issue. We adopt the value $r=-2$, producing with our potential an IVGQR centered at about $22-23 \mathrm{MeV}$, as seen from the middle plot of Fig. 1. 
This result agrees with experimental findings in several rare-earth nuclei [27] and more reliable data on ${ }^{208} \mathrm{~Pb}$ [24]. The theoretical E2 strength between 17 and $25 \mathrm{MeV}$, shown in the same plot and corresponding to the IVGQR with $K^{\pi}=1^{+}$, amounts to $1475 \mathrm{e}^{2} \mathrm{fm}^{4}$. The value is close to a rough estimate [7] from a schematic two-level model and agrees

qualitatively with recent results on the IVGQR in ${ }^{154} \mathrm{Sm}$, obtained from inelastic scattering of polarized protons [32]. The microscopic isoscalar coupling constant $k_{S}$ (5) gives rise to the isoscalar GQR with $K^{\pi}=1^{+}$at $11 \mathrm{MeV}$. Its E2 strength from the middle plot of Fig. 11, summed between 9 and $11 \mathrm{MeV}$, is $B(E 2)=1193 e^{2} f m^{4}$, in agreement with experiment [24,27.

\section{HIGH-ENERGY M1 STRENGTH AND $\left(e, e^{\prime}\right)$ CROSS SECTIONS}

The energy distribution of M1 strength in ${ }^{160} \mathrm{Gd}$, summed in bins of $1 \mathrm{MeV}$, is shown as histograms in Figs. 2 and 3 for different isovector ratios $r$ (6). The total and orbital M1 strengths are represented by contour lines and shaded areas, respectively. It is seen that the high-energy M1 strength is purely orbital, while the low-energy one contains spin contributions, which are dominant between 6 and $10 \mathrm{MeV}$. Comparison with the corresponding cases from Fig. [1 shows that the high-lying orbital M1 strength resides in the same energy region as the E2 IVGQR.

The low-lying orbital M1 strength is influenced only slightly when increasing the magnitude of the isovector interaction from $r=0$ to $r \approx-1$. A further increase $(r<-1)$ does not produce appreciable changes. The quadrupole interaction (2) contributes to the M1 e.w. sum-rule [1]. The repulsive isovector interaction shifts the high-lying orbital strength to higher energy and increases the e.w. sum.

Figure 2 displays results corresponding to the adopted isovector coupling constant, $r=$ -2. The lower plot of Fig. 2 was obtained by including only 2q.p. configurations below 20 $\mathrm{MeV}$ in the RPA calculations, as done also in [16]. A schematic, single excitation is produced at $22 \mathrm{MeV}$, carrying most of the high-energy strength: $B(M 1)=3.6 \mu_{N}^{2}, \quad B(E 2)=$ 
$1305 \mathrm{e}^{2} \mathrm{fm}^{4}$. However, this strongly collective state is no more present in more realistic calculations, including further 2q.p. configurations up to $30 \mathrm{MeV}$, as seen from the upper plot of Fig. 2. The single excitation is strongly fragmented over a broad energy region in this case.

Comparison with our previous results from Figs. 3 and 4 of Ref. [5] demonstrates that the strong fragmentation is not solely due to the large basis, but results also to a great extent from the restoration of rotational invariance. It is seen from these two figures that the quadrupole interaction alone produces a high-energy orbital M1 strength, which is still strongly concentrated in a narrow peak. In contrast, considerable fragmentation takes place after adding the symmetry-restoring interaction [1] (cases denoted by a prime in Figs. 3,4 of Ref. [5). This strong fragmentation is in a qualitative agreement with the present results, obtained from the rotationally invariant hamiltonian (6).

The summed M1 strength above $20 \mathrm{MeV}$ in the upper plot of Fig. 2 is equal to that of the single excitation at $22 \mathrm{MeV}$ from the bottom plot, resulting from the basis cut-off. The equality is due to the fact that the high-energy strength originates from 2q.p. configurations with $\Delta \mathcal{N}=2$, lying at about $15 \mathrm{MeV}$. Thus, configurations above $20 \mathrm{MeV}$ do not generate but only redistribute the high-energy strength, pushed by the repulsive isovector interaction from 15 to $22 \mathrm{MeV}$.

Let us consider the more realistic results in the upper plot of Fig. 2. The summed (predominantly orbital) M1 strength between 17 and $25 \mathrm{MeV}$ is $3.6 \mu_{N}^{2}$, in agreement with a recent estimate for ${ }^{154} \mathrm{Sm}$ from $\left(\vec{p}, \vec{p}^{\prime}\right)$ experiments [32]. It is derived from a sum-rule [33] connecting the M1 and E2 strengths. The strongest single $1^{+}$excitation from the considered energy region lies at $22.5 \mathrm{MeV}$ and carries only $0.25 \mu_{N}^{2}$ strength, i. e. it could be hardly resolved experimentally. This excitation overlaps only $4 \%$ with the microscopic scissors state [6.2], obtained by rotating the neutron and proton configurations of the deformed mean field in opposite directions. The low-lying $1^{+}$excitations below $9 \mathrm{MeV}$ overlap all together $77 \%$ with the scissors state, while the overlap of those between 17 and $25 \mathrm{MeV}$ amounts to 55\%. The total overlap of all the RPA excitations below $30 \mathrm{MeV}$ is 1.46 . Thus, the low- and 
high-lying excitations exhaust $53 \%$ and $38 \%$ of the total overlap with the scissors state, respectively.

Let us note that the total overlap is exactly $100 \%$ for a vanishig isovector interaction [2.5]. The isoscalar channel cancels the spurious (isoscalar) admixtures, but it does not contribute to the (purely isovector) scissors mode. Thus, an overlap of $100 \%$ is produced by the mean field alone. The overlap is not conserved in RPA since it corresponds to a non e.w. sum, while only energy-weighted sums are conserved in RPA. The excess beyond 100\% is generated by the isovector residual interaction, which is not present in the microscopic scissors state, constructed from the mean field alone. The overlap reaches $174 \%$ in the case $r=-3.6$, plotted in Fig. 3.

A more meaningful geometric interpretation of the orbital $1^{+}$excitations is provided by the comparison with low collective synthetic states [34]. A synthetic state is constructed for each RPA excitation by rotating out-of-phase neutron and proton configurations weighted with the RPA amplitudes of this excitation. A large overlap of a given RPA state with its synthetic counterpart is an indication for an isovector rotational motion, irrespectively of the low collectivity caused by the fragmentation. The strongest (low-energy orbital) M1 excitation overlaps usually $80-90 \%$ with its synthetic counterpart [2,21]. In contrast, no one of the strongest orbital states at high energy (around $22 \mathrm{MeV}$ in the upper plot of Fig. 2) overlaps more than $7 \%$ with its synthetic counterpart. The coherence is restored in the schematic single excitation at $22 \mathrm{MeV}$ in the lower plot: it overlaps almost $90 \%$ with its synthetic counterpart, confirming the presence of the scissors mode at high-energy.

It is clear from the above considerations that the scissors mode fragments over both the low- and high-energy orbital M1 excitations. This holds exactly for the isovector rotational model [17], formulated in the schematic two-level basis of the quantized deformed oscillator. It was found within this model that the scissors mode exhausts the whole non-spurious orbital M1 strength at low and high energy, corresponding to excitations with $\Delta \mathcal{N}=0,2$, respectively.

A strong isovector quadrupole interaction, based on the operators $\mathbf{Q}_{2, \pm 1}$, leads to similar 
results [5], apart from concentrating in a narrower energy region much more high-lying M1 strength. Most of it turns out to be spurious. After restoration of the rotational invariance, the high-lying strength is reduced and fragmented over a broad region [1, 5], in agreement with the present results, based on the rotationally invariant hamiltonian (6).

Results from different QRPA calculations are compared in Table I. Our results (first row) are similar to those obtained with a zero-range Landau-Migdal interaction 13, 14, (second row), apart from a lesser fragmentation in the latter case. This is seen on the example of ${ }^{164}$ Dy [14], where the strongest high-lying $1^{+}$state (at $21 \mathrm{MeV}$ ) has a large transition probability, $B(M 1)=1.26 \mu_{N}^{2}$. It seems that the concentration of strength is caused by the reduced basis of 2q.p. configurations adopted in Ref. [14]. As discussed above, the total strength is not influenced substantially by this procedure.

The results listed in the last row of Table I [16] are similar to those in the bottom plot of Fig. 3, because the same basis cut-off (20 MeV) and almost the same isovector ratio are used in both cases. The B(M1) value of the single excitation at $26 \mathrm{MeV}$ in the bottom plot of Fig. 3 is four times larger than the value $1.5 \mu_{N}^{2}$, listed in the last row of Table I. The difference is due to the fact that ${ }^{152} \mathrm{Dy}$, considered in [16], has a very small deformation, producing less orbital M1 strength than the well-deformed ${ }^{160} \mathrm{Gd}$, studied in the present work.

It is seen from the middle plot of Fig. 3 that a considerable fragmentation takes place after including all the 2q.p. configurations up to $35 \mathrm{MeV}$ in calculations. The strong collective excitation disappears and its strength is distributed over a wide energy range. As discussed in Sect. III, the present status of experiment on heavy deformed nuclei does not allow a reliable discrimination between the results in Figs. 2 and 3 , obtained with different isovector coupling constants.

Theoretical $\left(e, e^{\prime}\right)$ cross sections, calculated in DWBA 35] using our RPA wave functions, are plotted in Figs. 1 and 5. E2 (dotted curves) and M1 (dashed curves) contributions to the cross sections for a scattering angle $\theta=165^{\circ}$ are plotted versus the electron incident energy, together with their sum (continuous curves). The $\left(e, e^{\prime}\right)$ cross sections of the two strongest high-energy M1 transitions are plotted in Fig. 4. Case A corresponds to the RPA excitation 
with $E_{x}=21.55 \mathrm{MeV}$ and $B(M 1)=0.25 \mu_{N}^{2}$, while case C refers to the RPA excitation with $E_{x}=20.54 \mathrm{MeV}$ and $B(M 1)=0.20 \mu_{N}^{2}$. Although both states are composed mainly from configurations with $\Delta \mathcal{N}=2$, the leading 2q.p. components in the corresponding RPA wave functions are neutrons with $\Delta \mathcal{N}=4$ in the former state and protons with $\Delta \mathcal{N}=0$ in the latter state. Thus, the former excitation has a more pronounced volume character than the latter one.

In addition to the above two strongest excitations at high energy we consider also the two next strongest high-energy orbital M1 excitations from the upper plot of Fig. 2. These four excitations lie between 20.5 and $23 \mathrm{MeV}$, have a summed $B(M 1)$ value $0.73 \mu_{N}^{2}$, and a summed overlap with the scissors state $11 \%$. The sum of their cross sections is displayed

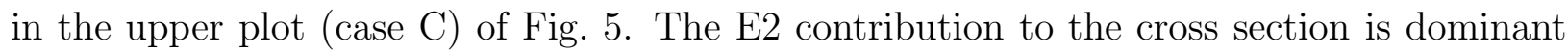
over the M1 cross section for incident energies below $100 \mathrm{MeV}$. The diffraction structure is smeared out, because the considered four excitations have very different transition densities.

The lower plot (case D) in Fig. 5 corresponds to the schematic collective excitation at 22 $\mathrm{MeV}$ from the lower plot of Fig. 2. It is seen from Fig. 5 that even in backward scattering the cross section is one order of magnitude larger for E2 than for M1 excitations. The displayed cross sections are calculated for $\theta=165^{\circ}$, an angle often used in experiments, but we have checked that the same E2 dominance is present even at a fully backward angle. This result is particularly interesting in view of the well-known expectation [36] for a strong enhancement of M1 over E2 excitations in backward scattering. Such a qualitative expectation is based on approximations (negligible excitation energy compared to the incident electron energy, PWBA), which do not hold for the DWBA cross section of the high-energy excitation, dispayed in Fig. 可.

The experimental resolution of single M1 excitations is hindered furthermore by the strong fragmentation at high energy. One should expect, therefore, that above $17 \mathrm{MeV}$ the experimental cross section of inelastically scattered electrons will originate almost exclusively from the E2 IVGQR and not from M1 transitions. In contrast, at low energy the M1 cross section dominates over the E2 cross section [37, at least for low transferred momenta. 


\section{CONCLUSIONS}

The high-lying M1 strength is almost purely orbital. It resides in the same energy region as the isovector E2 strength (IVGQR). Experimental data on the IVGQR in heavy deformed nuclei are scarce. The interpretation of the $\left(e, e^{\prime}\right)$ spectra rely on a rough estimate of the expected excitation energy of this resonance, based on the collective model. The main uncertainty arises from the poorly known isovector potential, which determines also the magnitude of the isovector coupling in the residual spin-independent interaction, used in microscopic calculations. We have adopted here a ratio $r=-2$ between the isovector and isoscalar coupling constants. The isoscalar constant is determined microscopically from the condition of rotational invariance. On the example of ${ }^{160} \mathrm{Gd}$, considered here, the isoscalar coupling gives rise to an isoscalar GQR at $11 \mathrm{MeV}$ with $B(E 2)=1193 e^{2} \mathrm{fm}^{4}$, in agreement with experiment.

The adopted isovector ratio, $r=-2$, produces in RPA an IVGQR whose $K^{\pi}=1^{+}$component is centered at about $22 \mathrm{MeV}$, but strongly fragmented over the interval 17-25 $\mathrm{MeV}$. Thus, even the strongest M1 transition from this region has a relatively small $B(M 1)$ value, $0.25 \mu_{N}^{2}$, in comparison with the strong low-lying orbital excitations. The high-lying strengths, summed in the above interval, are $B(E 2)=1475 e^{2} \mathrm{fm}^{4}, \quad B(M 1)=3.6 \mu_{N}^{2}$. The total RPA energy-weighted E2 strength exhausts exactly the classical sum rule for $K= \pm 1$.

The scissors mode is found to fragment over both the low- and high-energy orbital M1 excitations. Although the latter are more collective, the coherence of the scissors motion is destroyed at high energy by the increased fragmentation, due not only to the high level density, but also to the rotational invariance. Most of the strongly fragmented high-lying orbital excitations (17-25 MeV) are not performing well-pronounced rotational vibrations. This is seen from their small overlap with synthetic states, where such a geometrical motion is enforced by construction. Nevertheless, the orbital excitations from the above high-energy region have all together a large overlap, 55\%, with the collective scissors state constructed from the deformed mean field. The $1^{+}$excitations below $9 \mathrm{MeV}$ overlap $77 \%$ with the 
scissors state. The total scissors overlap of all the RPA excitations below $30 \mathrm{MeV}$ is 1.46. The excess above unity is produced by the isovector coupling, corresponding to a stronger attractive neutron-proton interaction, which enhances the scissors motion.

The recent canonical quantization of the isovector rotor in relative conjugate variables [17 provides simple analytical results in the deformed oscillator basis. They strongly support the interpretation of all the orbital M1 strength, at both low and high energies, as manifestation of the fragmented collective scissors mode.

The $\left(e, e^{\prime}\right)$ cross sections of $1^{+}$states above $17 \mathrm{MeV}$ originate mainly from E2 excitations even for backward scattering, while the contributions of the high-lying orbital M1 excitations to these cross sections are typically one order of magnitude smaller.

Thanks are due to Jochen Heisenberg for providing us with his DWBA code. This work is supported by the Deutsche Forschungsgemeinschaft (DFG). 


\section{REFERENCES}

[1] R. Nojarov and A. Faessler, Nucl. Phys. A484, 1 (1988).

[2] A. Faessler and R. Nojarov, Phys. Lett. B215, 439 (1988);

R. Nojarov and A. Faessler, Z. Phys. A336, 151 (1990);

R. Nojarov and A. Faessler, Mod. Phys. Lett. A13, 1171 (1993).

[3] N. Lo Iudice and F. Palumbo, Phys. Rev. Lett. 41, 1532 (1978); Nucl. Phys. A326, 193 (1979);

G. De Franceschi, N. Lo Iudice, and F. Palumbo, Phys. Rev. C 29, 1496 (1984).

[4] H. H. Pitz, U. E. P. Berg, R. D. Heil, U. Kneissl, R. Stock, C. Wesslborg, and P. von Brentano, Nucl. Phys. A492, 411 (1989);

W. Ziegler, N. Huxel, P. von Neumann-Cosel, C. Rangacharyulu, A. Richter, C. Spieler, C. De Coster, and K. Heyde, Nucl. Phys. A563, 366 (1993).

[5] R. Nojarov and A. Faessler, Nucl. Phys. A572, 17 (1994).

[6] A. E. L. Dieperink and E. Moya de Guerra, Phys. Lett. B189, 267 (1987);

E. Moya de Guerra, P. Sarriguren, and J. M. Udias, Phys. Lett. B196, 409 (1987).

[7] N. Lo Iudice and A. Richter, Phys. Lett. B228, 291 (1989); B304, 193 (1993);

N. Lo Iudice, F. Palumbo, A. Richter, and H. J. Wörtche, Phys. Rev. C 42, 241 (1990).

[8] T. Otsuka, Nucl. Phys. A507, 129c (1990).

[9] C. De Coester and K. Heyde, Nucl. Phys. A529, 507 (1991).

[10] T. Shimano and A. Ikeda, Progr. Theor. Phys. 88, 599 (1992);

A. Ikeda and T. Shimano, Nucl. Phys. A557, 573c (1993).

[11] I. Hamamoto and S. Åberg, Phys. Lett. B145, 163 (1984); Phys. Scripta 34, 697 (1986).

[12] C. Magnusson, Phys. Scripta 43, 460 (1991). 
[13] J. Speth and D. Zawischa, Phys. Lett. B211, 247 (1988); B219, 529 (1989).

[14] D. Zawischa and J. Speth, Z. Phys. A339, 97 (1990).

[15] D. Zawischa and J. Speth, Nucl. Phys. A553, 557c (1993).

[16] I. Hamamoto and W. Nazarewicz, Phys. Lett. B297, 25 (1992).

[17] R. Nojarov, Nucl. Phys. A571, 93 (1994).

[18] A. Faessler and R. Nojarov, Phys. Rev. C 41, 1243 (1990).

[19] R. Nojarov and A. Faessler, Phys. Rev. C 49, 213 (1994).

[20] M. I. Baznat and N. I. Pyatov, Yad. Fiz. 21, 708 (1975) [Sov. J. Nucl. Phys. 21, 365 (1975)].

[21] R. Nojarov, A. Faessler, P. Sarriguren, E. Moya de Guerra, and M. Grigorescu, Nucl. Phys. A563, 349 (1993).

[22] Y. Tanaka, Y. Oda, F. Petrovich, and R.K. Sheline, Phys. Lett. B83, 279 (1979).

[23] P. Sarriguren. E. Moya de Guerra, R. Nojarov, and A. Faessler, J. Phys. G 19, 291 (1993); J. Phys. G 20, 315 (1994).

[24] A. Van der Woude, Progr. Part. Nucl. Phys. 18, 217 (1987); Internat. Rev. Nucl. Phys. 7, 99 (1991).

[25] T. Suzuki and D. J. Rowe, Nucl. Phys. A289, 461 (1977).

[26] E. K. Warburton and J. Weneser, in Isospin in Nuclear Physics, ed. by D. H. Wilkinson (North-Holland, Amsterdam, 1969), p. 175.

[27] R. S. Hicks, I. P. Auer, J. C. Bergstrom, and H. S. Caplan, Nucl. Phys. A278, 261 (1977);

B. S. Dolbilkin, S. Ohsawa, Y. Torizuka, T. Saito, Y. Mizuno, and K. Saito, Phys. Rev. C 25, 2255 (1982). 
[28] P. Möller and J. R. Nix, Atomic Data and Nucl. Data Tables 39, 213 (1988).

[29] A. Bohr and B. R. Mottelson, Nuclear Structure (Benjamin, London, 1975), Vol. 2.

[30] T. Suzuki, Progr. Theor. Phys. 64, 1627 (1980).

[31] D. R. Bes, R. A. Broglia, and B. S. Nilsson, Phys. Reports C 16, 1 (1975).

[32] D. Frekers, H.-J. Wörtche, A. Richter, O. Häusser, R. S. Henderson, C. A. Miller, A. Trudel, M. C. Vetterli, and S. Yen, in Annual Report 1992/93 (Institut für Kernphysik, Universität Münster, Germany), p.43.

A. Richter, Darmstadt Preprints IKDA 94/10, 94/13, 94/15 (1994); Progr. Part. Nucl. Phys. 34, (in press) (1995).

[33] E. Moya de Guerra and L. Zamick, Phys. Rev. C 47, 2604 (1993).

[34] A. Faessler, R. Nojarov, and F. G. Scholtz, Nucl. Phys. A515, 237 (1990).

[35] J. Heisenberg and H. P. Blok, Ann. Rev. Nucl. Part. Sci. 33, 569 (1983).

[36] L. W. Fagg, Rev. Mod. Phys. 47, 683 (1975).

[37] R. Nojarov, A. Faessler, and M. Dingfelder, J. Phys. G 20, L111 (1994). 


\section{TABLES}

TABLE I. Comparison of different theoretical QRPA predictions on the summed high-energy M1( $\uparrow)$ strength and overlap with the scissors state $\mathbf{R}^{\dagger}|\rangle[2]$ in rare-earth nuclei.

\begin{tabular}{lcccc}
\hline \hline Nucleus & $\mathrm{E}$ & $\sum \mathrm{B}(\mathrm{M} 1)$ & $\sum \mathrm{EB}(\mathrm{M} 1)$ & $\sum\left|\left\langle\mathbf{R} 1^{+}\right\rangle\right|^{2}$ \\
& {$[\mathrm{MeV}]$} & {$\left[\mu_{N}^{2}\right]$} & {$\left[\mathrm{MeV} \mu_{N}^{2}\right]$} & $55^{b}$ \\
\hline${ }^{160} \mathrm{Gd}^{a}$ & $17-25$ & 3.6 & 76 & 30 \\
${ }^{164} \mathrm{Dy}^{c}$ & $21-23$ & 3.6 & 79 & 40 \\
${ }^{152} \mathrm{Dy}^{d}$ & 27 & 1.5 & 40 & \multirow{2}{*}{${ }^{c}$} \\
\hline \hline
\end{tabular}

${ }^{a}$ This work.

${ }^{b} 38 \%$ of the total overlap 1.46 .

${ }^{c}$ Ref. [14.

$d$ Ref. [16]. 


\section{FIGURES}

FIG. 1. Energy distribution of $B\left(E 2 ; 0_{\text {g.s. }}^{+} \rightarrow 2^{+} 1\right)$ values, summed in bins of $1 \mathrm{MeV}$, for ${ }^{160} \mathrm{Gd}$, corresponding to three different values of the isovector strength constant $k_{R}$ (6): $r=-0.6,-2.0,-3.6$.

FIG. 2. Energy distribution of $B\left(M 1 ; 0_{\text {g.s. }}^{+} \rightarrow 1^{+} 1\right)$ values summed in bins of $1 \mathrm{MeV}$ (contour histograms with a bright shading) for ${ }^{160} \mathrm{Gd}$, obtained with isovector strength ratio (6) $r=-2$. Dark-shaded areas: only orbital M1 strength. Bottom plot: only 2q.p. configurations below 20 $\mathrm{MeV}$ are included in calculations.

FIG. 3. The same as in Fig. 2, but for isovector strength ratios $r=-0.6,-3.6$. Basis cut-off in the bottom plot as in the lower plot of Fig. 2. Note the compressed ordinate scale in the bottom plot.

FIG. 4. DWBA $\left(e, e^{\prime}\right)$ cross sections of the strongest (case A) and the second strongest (case B) RPA excitations around $22 \mathrm{MeV}$ from the upper plot of Fig. 2 with $B(M 1)$ values 0.25 and 0.20 $\mu_{N}^{2}$, respectively. Scattering angle $\theta=165^{\circ}$. Contributions from E2 $\left(0_{\text {g.s. }}^{+} \rightarrow 2^{+} 1\right)$ (dotted curves) and $\operatorname{M1}\left(0_{\mathrm{g} . \mathrm{s} .}^{+} \rightarrow 1^{+} 1\right)$ (dashed curves) excitations of the same rotational band with $K^{\pi}=1^{+}$, and their sum (continuous curves) are plotted versus the electron incident energy.

FIG. 5. The same as in Fig. 1 , but for the summed cross sections of the four strongest M1 excitations around $22 \mathrm{MeV}$ from the top plot of Fig. 2 (case C) and the single state at $22 \mathrm{MeV}$ from the bottom plot of Fig. 2 (case D). 
Figure 1

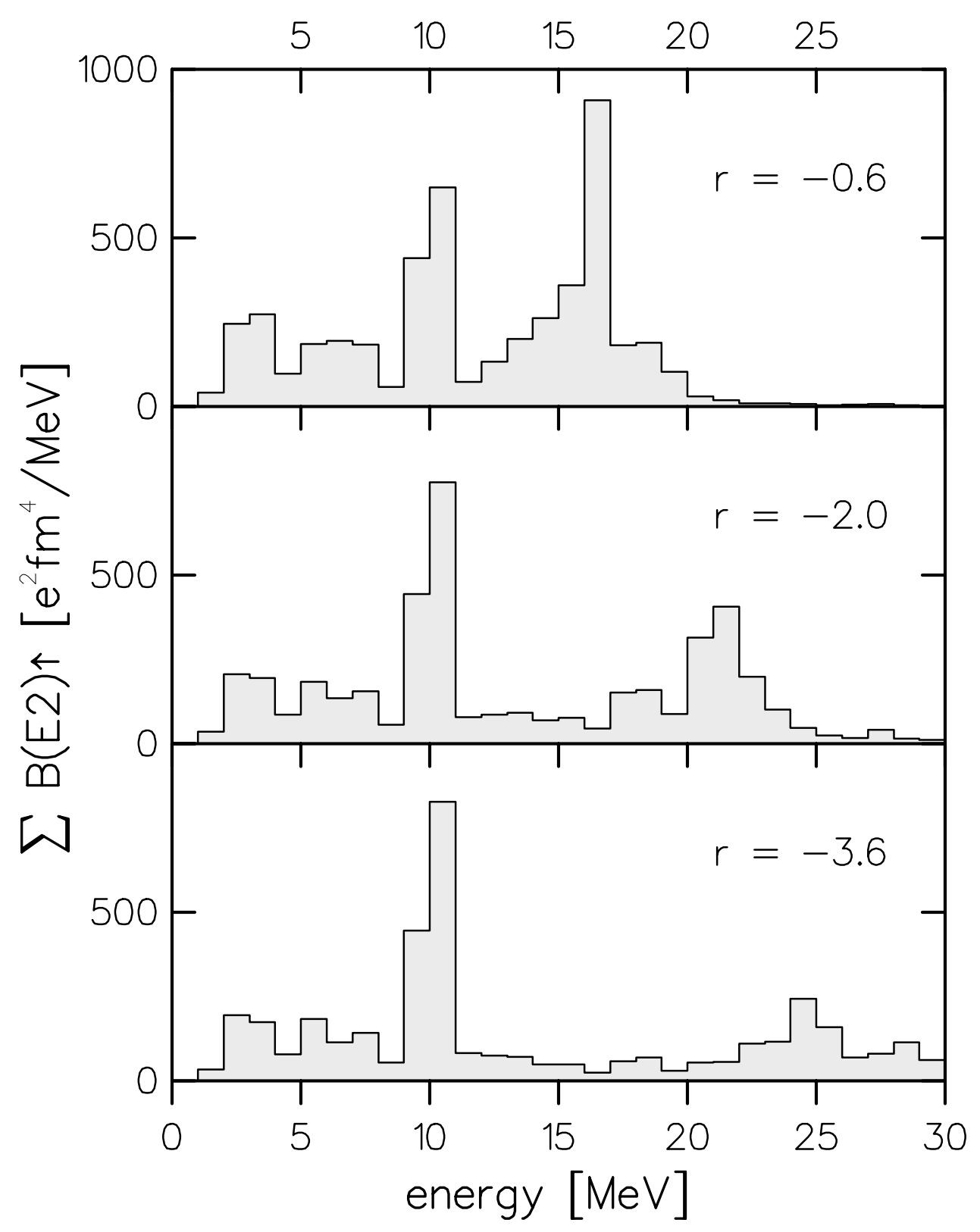


Figure 2

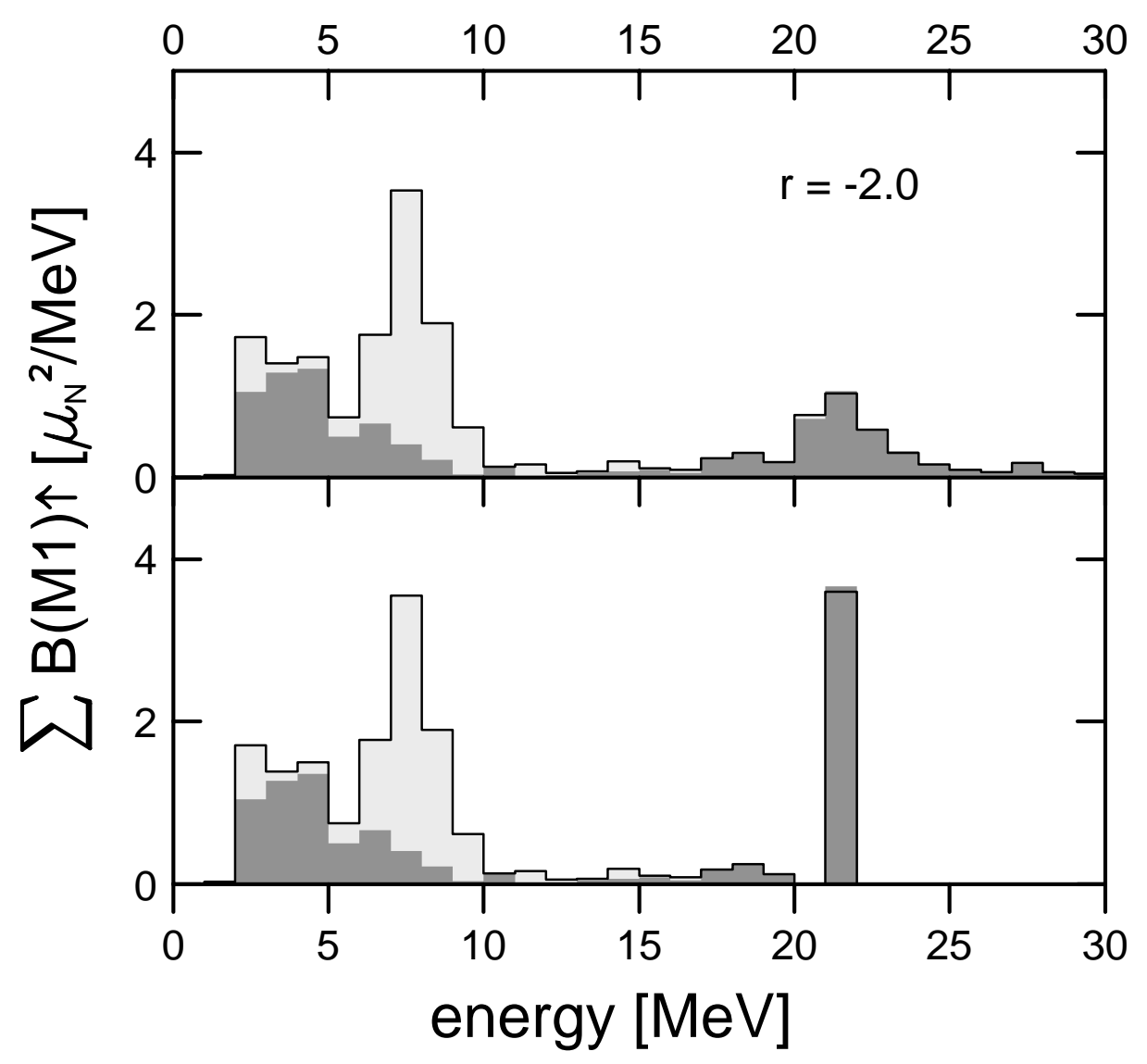


Figure 3

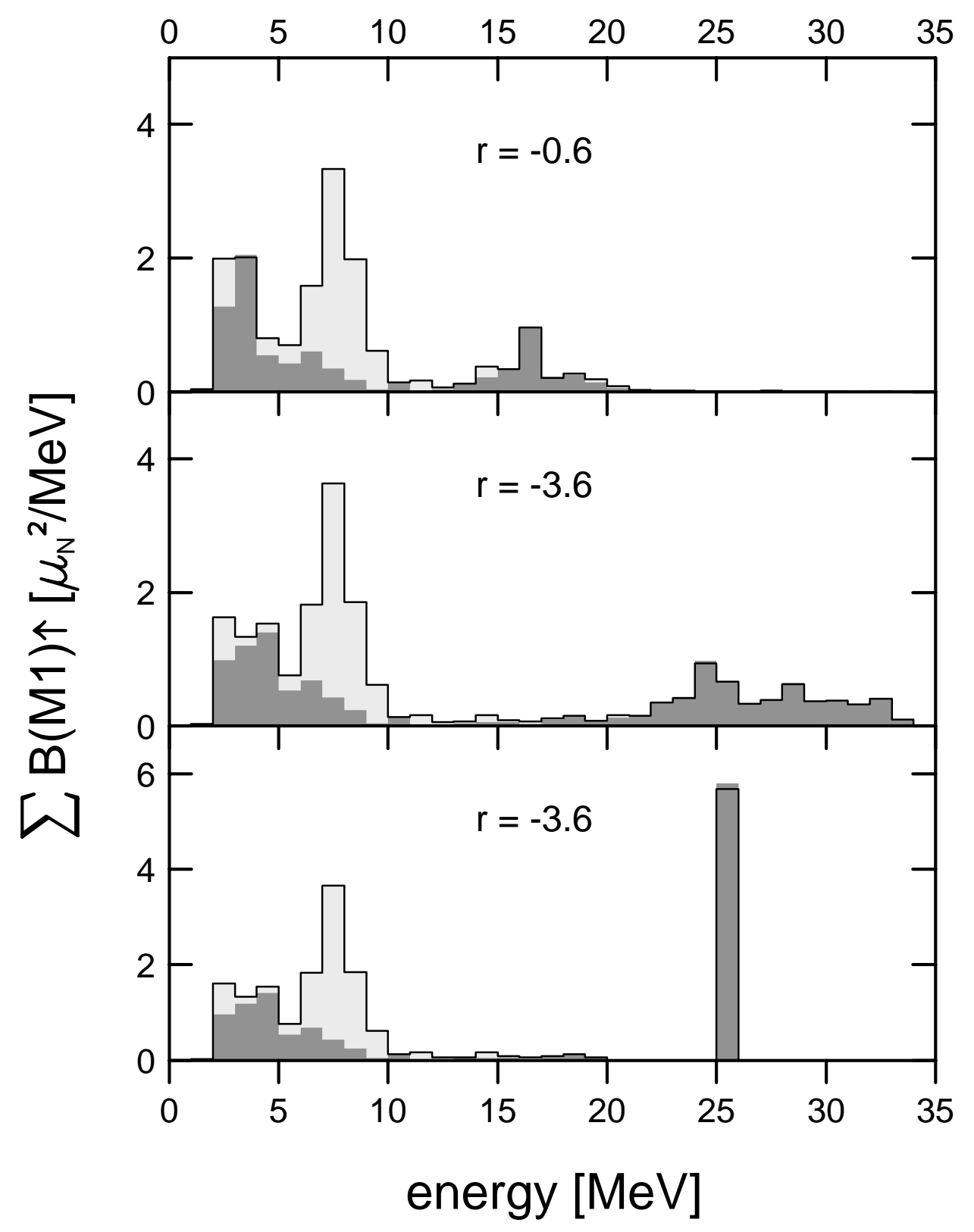


Figure 4

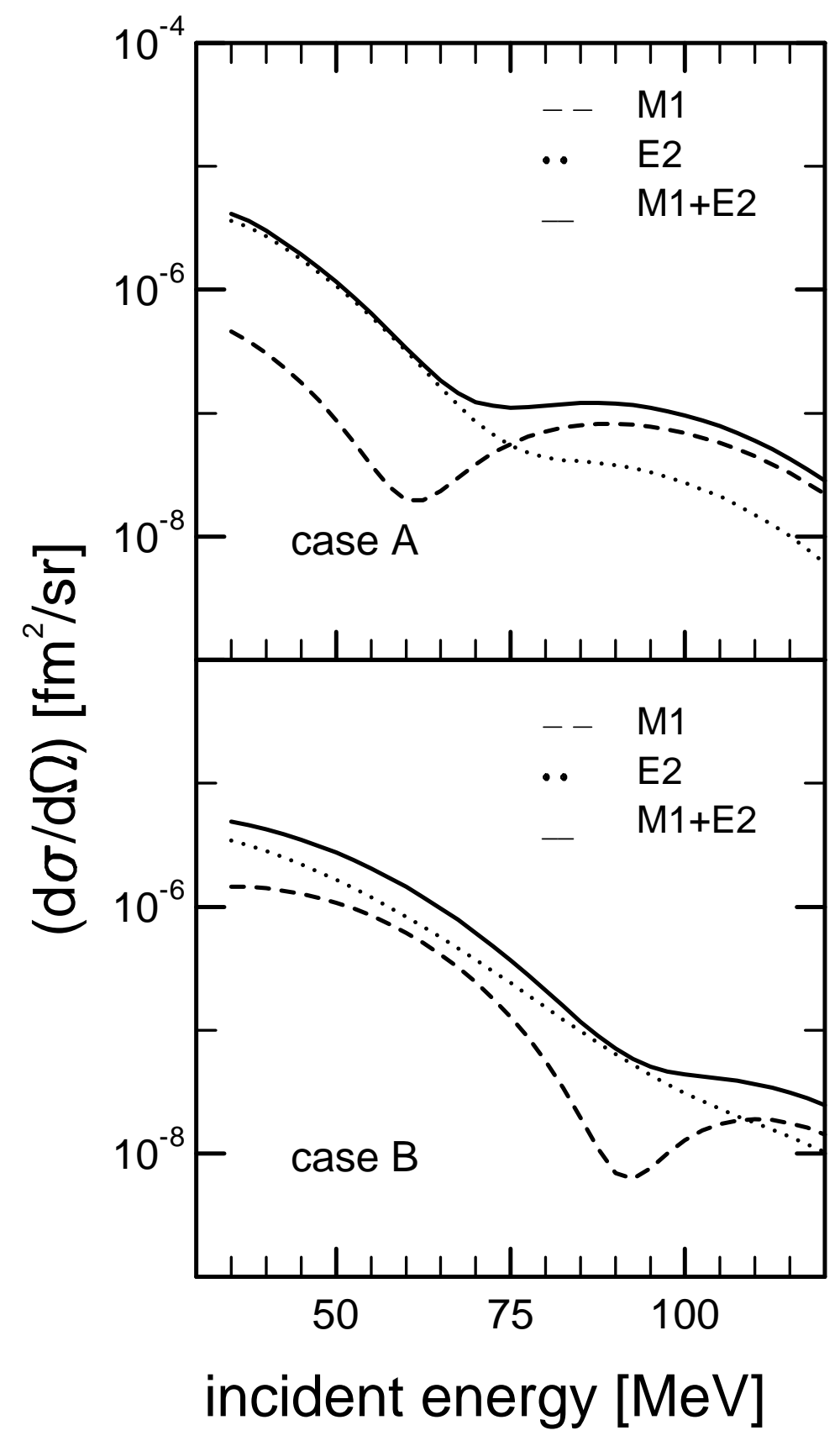


Figure 5

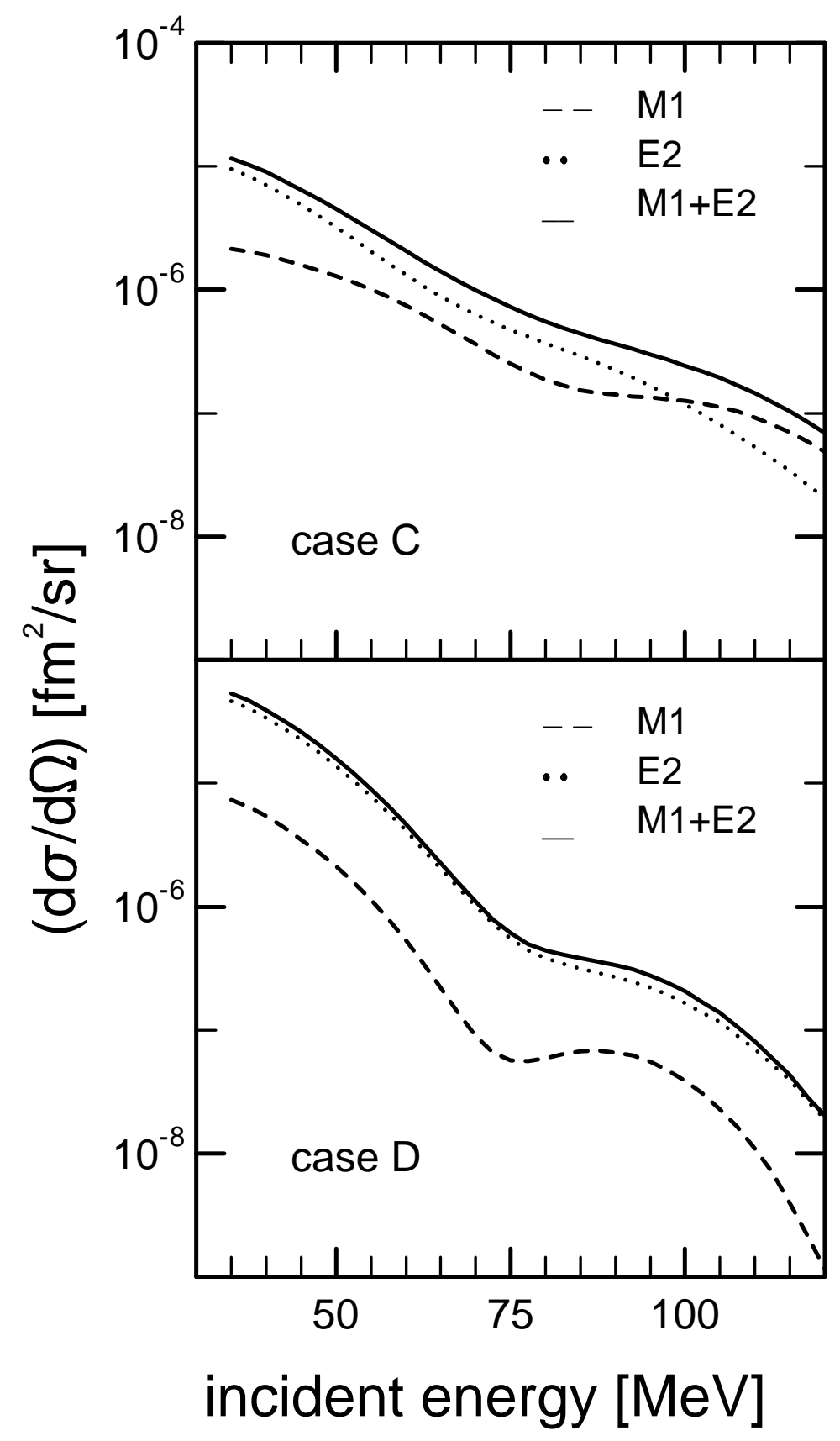

